\title{
Cambios del patrón de enfermedad en la postransición epidemiológica en salud en Chile, 1950-2003
}

\author{
Cecilia Luque ${ }^{1}$, Felipe A Cisternas², Magdalena Araya². \\ Changes in the patterns of disease \\ after the epidemiological transition \\ in health in Chile, 1950-2003
}

Background: During the twentieth century there was a change in the pattern of diseases in Europe, with an increase in the incidence of allergies and autoimmune disorders, that paralleled a decrease of infectious conditions. The Hygiene hypothesis proposes that these phenomena are causally related. Aim: To evaluate the epidemiological changes of allergic, autoimmune, and infectious diseases in Chile between 1950 and 2003. Material and methods: Search for the incidence and prevalence of these diseases in the national records published by the Ministry of Health, as well as through a systematic search of national literature using PubMed and Scielo as search engines. Results: The annual incidence of tuberculosis, rheumatic fever, measles, and typhoid fever has progressively diminished in Chile since 1970. Figures for the national prevalence for asthma, rheumatoid arthritis, and type I diabetes are scarce and difficult to compare, but clearly show an increasing epidemiological trend in the last 20 years. Conclusions: The national figures suggest that, although the country has only recently gone through an epidemiological transition in health problems, there are detectable changes that show the same trends described in Europe (Rev Méd Chile 2006; 134: 703-12).

(Key w ords: Autoimmune diseases; Chronic disease; Communicable diseases; Epidemiology)

Recibido el 11 de mayo, 2005. Aceptado el 23 diciembre, 2005.

Financiamiento: INTA, Universidad de Chile.

${ }^{1}$ Programa de especialización en Gastroenterología Pediátrica, Universidad de Chile. ${ }^{2}$ Instituto de Nutrición y Tecnología de los Alimentos (INTA), Universidad de Chile

A lo largo del siglo $\mathrm{XX}$, en Europa se tomó conciencia de la disminución de las enfermedades infecciosas al mismo tiempo que aumenta-

Correspondencia a: Magdalena Araya. Instituto de Nutrición y Tecnología de los Alimentos (INTA), Universidad de Chile. Macul 5540, Casilla 13811, Santiago, 11, Chile. Teléfono: 6781468. Fax: 2214030. E mail: maraya@inta.cl ban las enfermedades autoinmunes y alérgicas. Los estudios epidemiológicos y experimentales disponibles llevaron a la formulación de la hipótesis de la higiene», que propone que estos cambios estarían causalmente relacionados ${ }^{1,2}$. Ya en 1966, se postuló que el riesgo de esclerosis múltiple es mayor en las personas que pasan su niñez en un hogar con alto nivel de limpieza ${ }^{3}$; del mismo modo, se ha propuesto que las infecciones 
dentro del hogar a temprana edad tendrían un rol protector importante en el desarrollo de rinitis alérgica y de otras enfermedades alérgicas ${ }^{4}$. La hipótesis de la higiene propone que a lo lango de la evolución del hombre, la disminución progresiva de la exposición temprana a microorganismos, como la que se observa en países desarrollados, tiene como consecuencia cambios en la calidad de la flora indígena intestinal, con los cambios correspondientes en las funciones de barrera intestinal $^{5}$; de alguna manera, esto cambiaría las capacidades y tipos de respuesta inmune, que a su vez llevaría al aumento observado de las enfermedades atópicas y autoinmunitarias ${ }^{5}$.

Los mecanismos exactos según los cuales la hipótesis de la higiene explicaría las respuestas inmunes inadecuadas se desconocen, pero la evidencia experimental sugiere que la base estaría dada por un desequilibrio del balance de las respuestas Th1/Th2. La mayoría de los problemas autoinmunes involucran citoquinas producidas por células T del tipo helper 1 (Th1), mientras que las enfermedades alérgicas requieren citoquinas producidas por células T del tipo helper 2 (Th2). En condiciones fisiológicas, las citoquinas Th1 ejercen una regulación negativa sobre las células Th2 y, a su vez, las células Th1 responden a la acción de citoquinas Th2; estas reacciones proporcionan la base inmunológica inicial para que estas citoquinas estén implicadas en la protección contra la alengia y autoinmunidad después de diferentes cuadros infecciosos ${ }^{1,6}$. Cabe preguntar si en la hipótesis de la higiene, una infección específica 0 una serie de infecciones pueden cambiar permanentemente el tipo de respuesta inmune futura y si hay un rango de edades en el cual debe ocurrir la infección para producir este cambio.

Chile ha experimentado una transformación significativa de sus condiciones de salud, fundamentalmente debido a cambios demográficos, sociales y económicos. Este desarrollo está asociado a una creciente industrialización y urbanización, la cual se ha traducido en avances tecnológicos y una mejoría en la atención médi$\mathrm{ca}^{7}$. Es interesante plantearse si en la situación postransicional en salud que se describe actualmente en el país, se replica lo sucedido en países desarrollados, en términos de que se haya producido una disminución de las enfermedades infecciosas y un aumento de las enfermedades autoinmunes y alérgicas. Este estudio tuvo por objetivo evaluar el perfil epidemiológico de patologías frecuentes en estos dos tipos de enfermedades, revisando la información del país publicada entre los años 1950 y 2003.

\section{MATERIALES Y MÉTODOS}

Este es un estudio epidemiológico descriptivo de la incidencia y prevalencia de enfermedades infecciosas, autoinmunes y alérgicas de los últimos 50 años, en Chile. Para obtener los datos nacionales de incidencia de enfermedades infecciosas entre 1950 y 2003, se revisaron los Anuarios de Enfermedades de Notificación Obligatoria de la biblioteca del Ministerio de Salud (MINSAL), al igual que los boletines electrónicos disponibles del MINSAL (El Vigía).

No existen datos equivalentes sobre la epidemiología de enfermedades autoinmunes y alérgicas, debido a que éstas no son de declaración obligatoria y a que existen pocas investigaciones nacionales publicadas en estos temas. Se revisó sistemáticamente la literatura nacional e internacional en el período 1950-2003, que refirieran datos de población chilena, mediante los programas PubMed y Scielo, utilizando como criterios de búsqueda las palabras claves: asma, diabetes, diabetes mellitus tipo 1 (DM1), enfermedad de Crohn, artritis reumatoide y esclerosis múltiple. En la elección de las enfermedades escogidas para la comparación, se tomó en cuenta aquellas que aparecen en otros estudios de este tipo, pero, en gran parte, la decisión final estuvo basada en la disponibilidad de datos. Se aceptaron todos los artículos publicados, sin límites de sexo, edad, lenguaje o tipo de publicación. Se encontraron dieciocho artículos sobre DM1, ocho de enfermedad reumática, siete de asma, uno de esclerosis múltiple, pero ninguna publicación epidemiológica nacional sobre enfermedad de Crohn. Se revisaron todos los artículos encontrados, evaluando si 1) incluían información sobre incidencia o prevalencia de las distintas enfermedades definidas y 2) si presentaban una metodología clara, explícita y comparable. A continuación se presenta el análisis efectuado en un total de siete publicaciones (Tabla 1) que cumplían con los requisitos mencionados, incluyendo cuatro sobre asma, dos sobre enfermedad reumática y uno sobre DM1. 
Tabla 1. Resumen de artículos sobre asma, artritis reumatoide y D M 1 seleccionados, correspondientes al período 1950-2003

\begin{tabular}{|c|c|c|c|}
\hline Condición & Tipo de estudio & Metodología & Referencia \\
\hline $\begin{array}{l}\text { Asma } \\
\text { Escolares }\end{array}$ & $\begin{array}{l}\text { Encuesta clínico-epidemiológica a padres } \\
\text { de } 2.759 \text { niños en } 6 \text { colegios de Santiago }\end{array}$ & $\begin{array}{l}\text { Recolectó información referida a: } \\
\text { a) antecedente de síntomas obstructivos, bronquitis } \\
\text { obstructiva, asma bronquial } \\
\text { b) prevalencia en el último año } \\
\text { c) prevención de otras afecciones crónicas }\end{array}$ & $S$ \\
\hline $\begin{array}{l}\text { Asma } \\
\text { Escolares }\end{array}$ & $\begin{array}{l}\text { Muestra aleatoria de } 3.000 \text { escolares de } \\
\text { Santiago entre 6-7 y } 13-14 \text { años }\end{array}$ & $\begin{array}{l}\text { Metodología estandarizada validada: ISAAC } \\
\text { Se recolectó información referida a: } \\
\text { a) sibilancias alguna vez } \\
\text { b) asma alguna vez } \\
\text { c) sibilancias últimos } 12 \mathrm{~m} \\
\text { d) frecuencia episodios de sibilancias } \\
\text { e) episodio severo de sibilancias últimos } 12 \mathrm{~m} \\
\text { f) sibilancias con ejercicios últimos } 12 \mathrm{~m} \\
\text { g) despertar en noche por sibilancias } \\
\text { h) despertar nocturno por ataques de tos seca } \\
\text { en últimos } 12 \mathrm{~m}\end{array}$ & 9 \\
\hline $\begin{array}{l}\text { Asma } \\
\text { Pobl. General }\end{array}$ & $\begin{array}{l}\text { Muestra aleatoria en } 412 \text { hogares de } \\
\text { Santiago con } 2.074 \text { personas }\end{array}$ & Cuestionario precodificado personal y familiar & 10 \\
\hline $\begin{array}{l}\text { Asma } \\
\text { Pobl. General }\end{array}$ & $\begin{array}{l}\text { Muestra aleatoria en } 1.000 \text { viviendas } \\
\text { de Santiago con } 4.700 \text { personas }\end{array}$ & Cuestionario estructurado personal y familiar & 11 \\
\hline $\begin{array}{l}\text { Artritis } \\
\text { Reumatoide } \\
\text { Pobl. General }\end{array}$ & $\begin{array}{l}\text { Registros de mortalidad y morbilidad } \\
\text { del Servicio Nacional de Salud y diversos } \\
\text { consultorios y hospitales }\end{array}$ & Registros nacionales del Servicio Nacional de Salud & 12 \\
\hline $\begin{array}{l}\text { Artritis } \\
\text { Reumatoide } \\
\text { Pobl. General }\end{array}$ & $\begin{array}{l}\text { Muestra aleatoria de } 2 \text { censos, } 1970 \text { y } 1982 \text {, } \\
\text { con } 2.074 \text { y } 2.820 \text { personas respectivamente }\end{array}$ & $\begin{array}{l}\text { Encuesta de morbilidad y atención médica } \\
\text { en Santiago }\end{array}$ & 13 \\
\hline $\begin{array}{l}\text { DM1 } \\
\text { Niños 6-14 } \\
\text { años }\end{array}$ & $\begin{array}{l}\text { Registro estandarizado de captura y } \\
\text { recaptura decasos de } 2 \text { fuentes: 1) registros } \\
\text { médicos en hospitales y clínicas privadas, } \\
\text { diabetólogos y pediatras; 2) registro de la } \\
\text { Fundación de Diabetes Juvenil }\end{array}$ & $\begin{array}{l}\text { Metodología Diamond con los siguientes criterios } \\
\text { de inclusión: Edad de inicio de 0-14 años, } \\
\text { diagnóstico de diabetes en el periodo de estudio, } \\
\text { uso de insulina desde el inicio de la enfermedad, ser } \\
\text { residente de la región metropolitana de Santiago } \\
\text { al momento del diagnóstico }\end{array}$ & r \\
\hline
\end{tabular}

\section{RESUlTADOS}

Los datos obtenidos de los Anuarios de Enfermedades de Notificación Obligatoria, MINSAL, para los últimos 50 años, demuestran un descenso gradual en la incidencia de enfermedades infecciosas de declaración obligatoria (Tabla 2). Para graficar las frecuencias relativas de las cuatro enfermedades analizadas, se construyó un índice asignando $100 \%$ al valor más alto para cada enfermedad (Figura 1). En esta Figura se incluyeron los datos desde el año 1978, debido a que la tendencia al descenso es más clara desde esa fecha, mientras que en el período 1950-1977 (Tabla 2) las incidencias presentaban gran variabilidad. 
Tabla 2. Incidencia de enfermedades infecciosas por 100.000 habitantes por año. Fuente: Anuarios de Enfermedades de N otificación O bligatoria M IN SAL 1950 al 2003

\begin{tabular}{|c|c|c|c|c|}
\hline Año & Sarampión & Tifoidea & Tuberculosis & Fiebre reumática \\
\hline 1950 & 18 & 55,8 & - & - \\
\hline 1951 & 60,3 & 68,1 & - & - \\
\hline 1952 & 66,6 & 81,2 & - & - \\
\hline 1953 & 114,6 & 66 & - & - \\
\hline 1954 & 100,7 & 61,3 & - & - \\
\hline 1955 & 174,6 & 65,6 & - & - \\
\hline 1956 & 155,4 & 64,9 & - & - \\
\hline 1957 & 177,4 & 75,7 & - & - \\
\hline 1958 & 389,9 & 64,6 & - & - \\
\hline 1959 & 196,3 & 44,8 & - & - \\
\hline 1960 & 429,7 & 59,7 & - & - \\
\hline 1961 & 493,2 & 59,2 & - & - \\
\hline 1962 & 471 & 48,5 & - & - \\
\hline 1963 & 348,5 & 51,1 & - & - \\
\hline 1964 & 428,6 & 56,4 & - & - \\
\hline 1965 & 152,8 & 65,3 & - & - \\
\hline 1966 & 269,2 & 52,2 & - & - \\
\hline 1967 & 175,3 & 50,6 & - & - \\
\hline 1968 & 78,2 & 77,6 & - & - \\
\hline 1969 & 102,3 & 57,5 & - & - \\
\hline 1970 & 232,8 & 56,2 & - & - \\
\hline 1971 & 182,6 & 49,4 & - & - \\
\hline 1972 & 63,9 & 46 & - & - \\
\hline 1973 & 38,6 & 36,8 & - & - \\
\hline 1974 & 165 & 45,7 & - & - \\
\hline 1975 & 81,3 & 59 & - & - \\
\hline 1976 & 24,2 & 58,8 & - & - \\
\hline 1977 & 10 & 108,2 & - & . \\
\hline 1978 & 142,2 & 121,2 & - & 2,2 \\
\hline 1979 & 313,3 & 98 & - & 3,2 \\
\hline 1980 & 34,5 & 97,6 & - & 1,4 \\
\hline 1981 & 57,6 & 95,2 & - & 1,6 \\
\hline 1982 & 81,9 & 111,1 & 60,4 & 2,4 \\
\hline 1983 & 57,6 & 119,8 & 59,8 & 3 \\
\hline 1984 & 40,1 & 76,6 & 55,2 & 2,5 \\
\hline 1985 & 141,1 & 62,4 & 55 & 2 \\
\hline 1986 & 101,9 & 60,6 & 56,5 & $1, \overline{9}$ \\
\hline 1987 & 20,9 & 45,1 & 50 & 1,3 \\
\hline 1988 & 353,6 & 40,6 & 49,6 & 1 \\
\hline 1989 & 100,4 & 50,7 & 51,9 & 1 \\
\hline 1990 & 14,9 & 39,3 & 46,7 & 0,6 \\
\hline 1991 & 15,8 & 37,4 & 41,3 & 0,5 \\
\hline 1992 & 2,9 & 14 & 39,2 & 0,5 \\
\hline 1993 & 0 & 11,9 & 33,4 & 0,3 \\
\hline 1994 & 0 & 11,2 & 29,6 & 0,2 \\
\hline 1995 & 0 & 10 & 29,2 & 0,2 \\
\hline 1996 & 0 & 9,7 & 29 & 0,1 \\
\hline 1997 & 0,4 & 8,5 & 26,5 & 0,1 \\
\hline 1998 & 0 & 7,6 & 24,6 & 0 \\
\hline 1999 & 0,2 & 5,3 & 22,6 & - \\
\hline 2000 & 0 & 5,8 & 19,8 & - \\
\hline 2001 & 0 & 4,9 & 20,2 & - \\
\hline 2002 & 0 & 4,7 & 19,7 & - \\
\hline 2003 & 0 & 3,7 & 19,4 & - \\
\hline
\end{tabular}




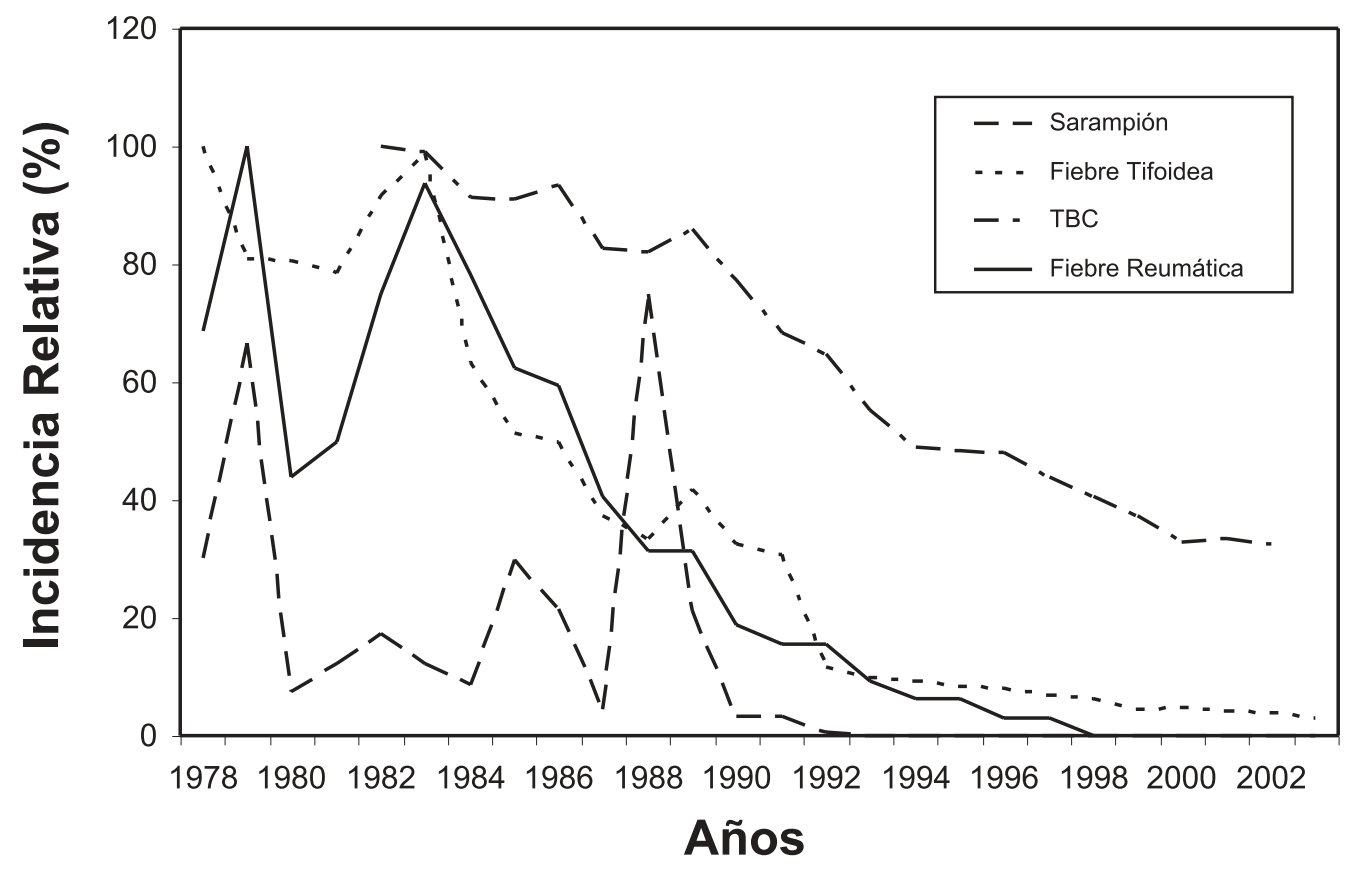

Figura 1. Incidencia relativa de las enfermedades infecciosas seleccionadas, en Chile, 1978-2003.

La incidencia de sarampión disminuyó de una tasa de 493,2/100.000 habitantes en 1961, a cero en 2003. Los casos de fiebre tifoidea llegaron a su máximo punto en 1978, con 121,2/100.000 habitantes y han bajado gradualmente hasta la tasa de 3,7/ 100.000 habitantes en 2003. Similarmente, la incidencia de fiebre reumática disminuyó de una tasa de sobre 3/100.000 habitantes en el año 1979, a cero en 1998, último año en el cual se consignó esta enfermedad en el MINSAL. Finalmente, los casos de tuberculosis han tenido un descenso gradual $\mathrm{y}$ sostenido, pasando de 60,4/100.000 habitantes en 1982 a 19,4/100.000 en 2003 (Tabla 2).

La incidencia de DM1 en niños y niñas de Santiago ${ }^{14}$ ha aumentado progresivamente en los últimos 18 años (Figura 2). Específicamente, la tasa anual aumentó entre 1986 y 2003, de 2,05/ a $8,47 / 100.000$ y de 3,11/ a 6,61/100.000 en niños y niñas, respectivamente (Tabla 3). Existen pocos estudios epidemiológicos chilenos comparables de asma y artritis reumatoide (Tabla 4). Se encon- traron dos estudios de asma en escolares con metodología similar, que muestran una prevalencia ascendente entre 1979 y 1996 de 5,4\% a 10,1\% y de $4,2 \%$ a $11,9 \%$ en escolares de 6 a 8 años y de 12 a 14 años, respectivamente. En estudios poblacionales generales, se observó un aumento de la prevalencia de asma, de 0,2\% a 1,4\% entre los años 1977 y 1994 (Tabla 4). Por su parte, la prevalencia de artritis reumatoide en la población general, se ha incrementado gradualmente de 0,4\% a 1,8\% entre 1963 y 1983 (Tabla 4).

\section{DisCUSIÓN}

Los datos analizados sugieren que en Chile los cambios epidemiológicos con disminución de patologías infecciosas versus el aumento de las relacionadas a una respuesta inmune inadecuada, presentan una tendencia similar a la descrita en países desarrollados. Aunque el fenómeno encon- 


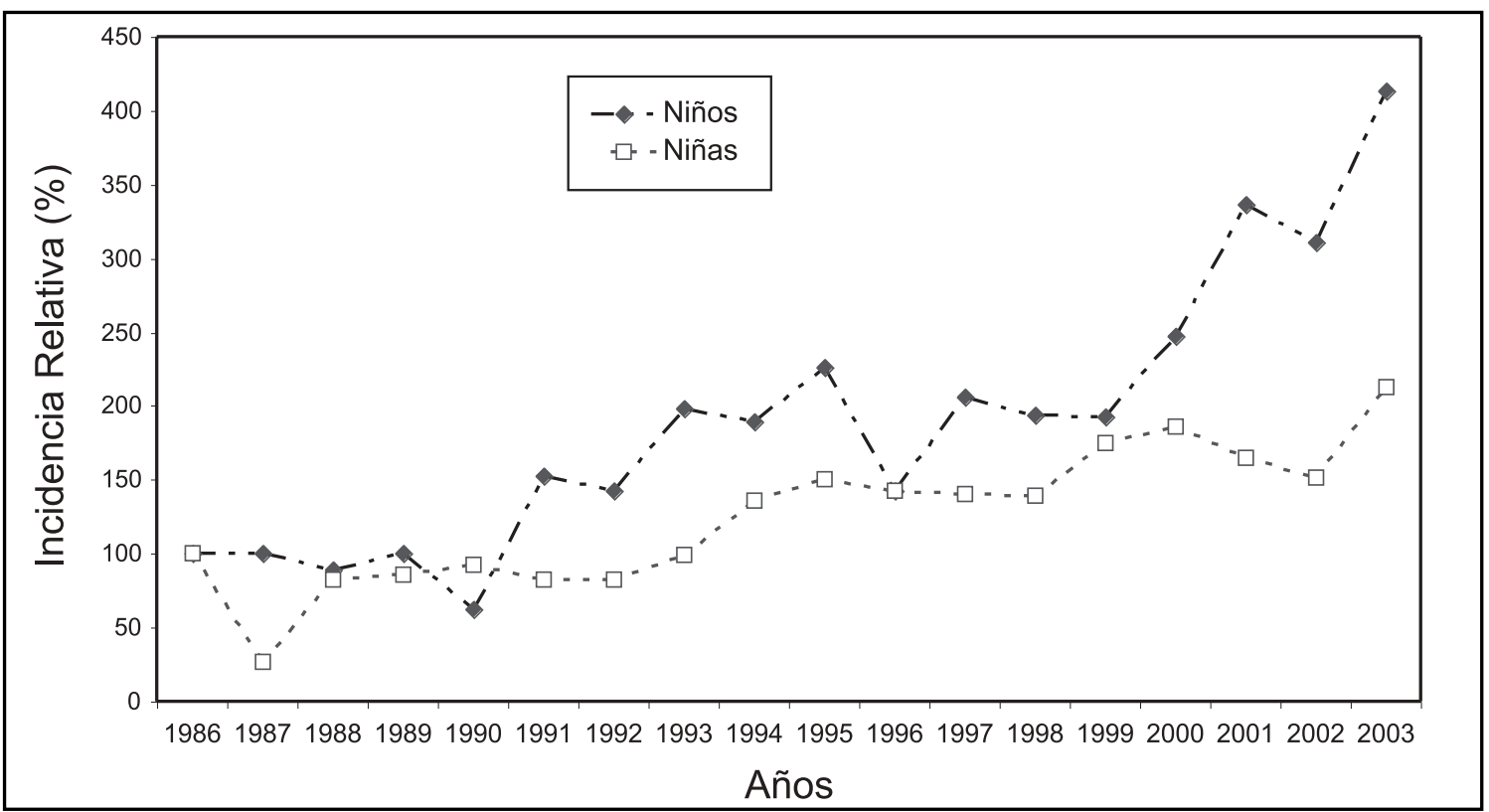

Figura 2. Incidencia relativa de DM1 en niños y niñas en Chile, 1986-2003.

Tabla 3. Incidencia de D M 1 en niñosy niñas de Santiago entre 1986-2003 por 100.000 habitantes $^{14}$

\begin{tabular}{|lll|}
\hline Año & Niños & Niñas \\
\hline 1986 & 2,05 & 3,11 \\
1987 & 2,05 & 0,83 \\
1988 & 1,83 & 2,57 \\
1989 & 2,05 & 2,66 \\
1990 & 1,27 & 2,88 \\
1991 & 3,12 & 2,57 \\
1992 & 2,93 & 2,57 \\
1993 & 4,07 & 3,09 \\
1994 & 3,88 & 4,24 \\
1995 & 4,63 & 4,66 \\
1996 & 2,93 & 4,43 \\
1997 & 4,22 & 4,38 \\
1998 & 3,97 & 4,32 \\
1999 & 3,96 & 5,45 \\
2000 & 5,08 & 5,80 \\
2001 & 6,90 & 5,13 \\
2002 & 6,38 & 4,72 \\
2003 & 8,47 & 6,61 \\
\hline
\end{tabular}

trado es interesante, hay que tener cautela al sacar conclusiones, porque en nuestro país las estadísticas sobre enfermedades infecciosas tienen representatividad nacional y han sido de buena calidad durante todo el período analizado; en cambio, los datos sobre enfermedades no infecciosas no son comparables, ya que no hay registros nacionales para las patologías incluidas en este trabajo. Los datos se refieren a estudios limitados, con tamaños muestrales más bien pequeños y en determinadas edades. Otro aspecto que debe tomarse en cuenta es que algunos criterios de definición de patologías (por ejemplo asma y diabetes) han cambiado en el curso del tiempo. Estos dos aspectos constituyen la principal limitación del presente trabajo; sin embargo, junto con esto, debemos decir que los estudios que se incluyeron en el análisis tenían una metodología adecuada y las tendencias de todas las condiciones evaluadas fueron semejantes y claras. El hecho que algunas definiciones de patologías cambien a lo largo del tiempo no es modificable y afecta a casi todos los análisis de este tipo que se han publicado; en nuestro caso, hemos indicado los datos en que este factor puede estar influyendo para alertar al lector acerca del problema. 
Tabla 4. Prevalencia (\%) de asma en escolares y población general (1977-1999) y artritis reumatoide en población general (1963-1983)

\begin{tabular}{|lllcc|}
\hline Patología & Año estudio & \multicolumn{1}{c}{ Edades } & Prevalencia & Referencia \\
\hline Asma escolares & 1979 & 6-8 años & 5,2 & 8 \\
& & 12-14 años & 4,2 & 9 \\
Asma escolares & $1992-1996$ & 6-7 años & 10,1 & 11,95 \\
(Estudio ISAAC) & & 13-14 años & 0,2 & 10 \\
Asma total & 1977 & Población general & 1,4 & 11 \\
Asma total & $1993-1994$ & Población general & 0,4 & 12 \\
Artritis reumatoide & $1963-1964$ & Población general & 1,22 & 13 \\
Artritis reumatoide & 1977 & Población general & 1,8 & \\
& 1983 & & & \\
\hline
\end{tabular}

La información nacional sobre enfermedades infecciosas muestra una clara disminución en la incidencia a través del tiempo, similar a lo observado en estudios europeos y norteamericanos ${ }^{1,5,15}$. El fenómeno obedece a múltiples factores, entre los que cabe destacar el uso efectivo de antibióticos, de un programa de vacunación eficiente de alta cobertura y de una mejoría en el saneamiento ambiental, higiene general y condiciones socioeconómicas.

En el aumento reciente de las enfermedades atópicas y autoinmunes en los países desarrollados, se reconoce la participación de un conjunto de factores genéticos, ambientales y socioeconómicos. Los que sufren de estas enfermedades portan rasgos genéticos que los predisponen a ellas. Por ejemplo en Japón, los alelos HLA que aumentan el riesgo de desarrollar DM1 tienen muy baja frecuencia y esto se traduce en una incidencia más reducida de DM1 entre los japoneses en comparación con poblaciones caucásicas $^{16}$. Similarmente, la frecuencia de concordancia entre gemelos monozigotos para DM1 (40\%) y asma (75\%), demuestra claramente una predisposición genética para estas enfermedades $^{17,18}$. Sin embargo, la genética no logra explicar la totalidad de los cambios epidemiológicos observados, ya que se han producido a una velocidad no compatible con la aparición de nuevas mutaciones. Es probable que también sean importante cambios en la exposición a ciertos factores ambientales como virus, bacterias, aeroalergenos contaminantes y nutrientes ${ }^{1,5}$. Un buen ejemplo de la influencia de este tipo de factores, es la similitud en la frecuencia de nuevos casos de DM1 que los hijos de inmigrantes pakistaníes en el Reino Unido tienen con la población local, la cual es 10 veces más alta que la incidencia de DM1 en Pakistán ${ }^{19}$.

Es interesante analizar la asociación de enfermedades atópicas y autoinmunitarias a partir de una disminución en infecciones. Se sabe que la respuesta inmune está modulada por los fenotipos de tipo Th1 y Th2, mediados por citoquinas específicas para cada tipo de respuesta y que normalmente están controlados entre ellos. Cuando predomina uno de ellos, por ejemplo, cuando existe el fenotipo asociado con un aumento de la respuesta Th2, generalmente, se observa sensibilización y enfermedades atópicas, como eczema 0 asma $^{20,21}$. Por su parte, las respuestas de tipo Th1, generalmente provocadas por patógenos microbianos intracelulares, regulan negativamente la inmunidad de tipo Th2. Así, el menor contacto con microorganismos y menor ocurrencia de enfermedades infecciosas a temprana edad conduciría a una respuesta mitigada en la dirección Th1, resultando en un predominio de la respuesta Th2 ${ }^{5}$. Este argumento se ha usado para explicar el predominio de glomerulonefritis membrano proliferativa en países en desarrollo, en comparación a las glomerulonefritis de otros tipos que predominan en países desarrollados ${ }^{22}$, algunas enfermedades respiratorias ${ }^{23}$, enfermedad inflamatoria intestinal cró- 
nica ${ }^{24}$, así como también en la respuesta a algunas vacunas, como la $\mathrm{BCG}^{25}$. Otro ejemplo interesante es la protección a la atopia conferida por la seropositividad al virus de la hepatitis $\mathrm{A}^{26}$. Las células $\mathrm{T}$ expresan un gen (TIM-1) durante el desarrollo de las respuestas Th2 que regula la producción de citoquinas. Este gen es, además, uno de los receptores para el virus de la hepatitis A, lo que sugiere que el efecto protector de esta infección resulta de una inmunomodulación directa desde el contacto con el virus. La demostración experimental de este tipo de fenómenos es necesaria para validar la hipótesis de la higiene.

Resulta interesante analizar la incidencia de la diabetes. Actualmente hay consenso en que la prevalencia global de diabetes está aumentan$\mathrm{do}^{27,28}$, pero es importante destacar que en este análisis debe entrar sólo la DM1. El último estudio epidemiológico chileno de DM1 ${ }^{14}$ demuestra un aumento constante de casos nuevos en los últimos 18 años. Estos datos están corroborados por informes previos de prevalencia e incidencia de DM1 por los mismos autores ${ }^{29}$. Sin embargo, llama la atención que, aunque vaya en aumento, la tasa nacional está por debajo de la de países desarrollados y sobre todo de España ${ }^{30}$. Esto podría explicarse por la etnicidad de la población chilena, la cual tiene un origen español importante, mezclado con grupos autóctonos (principalmente mapuche). Estudios previos han demostrado que la incidencia de DM1 en mapuches, no sólo es significativamente inferior a la del resto del país, sino una de las más bajas del mundo ${ }^{31}$ y esto podría explicar, al menos parcialmente, las tasas nacionales más bajas. Será importante continuar la evaluación epidemiológica de DM1 en los diferentes grupos étnicos del país para determinar si también ellos siguen el patrón al alza de los últimos años.

Los cuatro estudios de prevalencia de asma en Santiago que hemos incluido en el análisis, demuestran un aumento en el número de personas afectadas, tanto en jóvenes, como en la población general. Resulta muy difícil comparar los datos publicados sobre asma, ya que a lo largo del tiempo en el mundo han cambiado significativamente las definiciones y las metodologías usadas. Los estudios ISAAC (International Study of Asthma and Allergies in Childhood) han intentado evitar este problema utilizando métodos estandarizados $^{32}$. Los últimos datos chilenos muestran una alta prevalencia de síntomas respiratorios relacionados con asma en los niños ${ }^{9}$. Se ha postulado que el aumento de los contaminantes atmosféricos estarían implicando en el aumento de las manifestaciones clínicas, más que en la incidencia del asma bronquial ${ }^{33,34}$. Datos publicados por Corvalán y cols (2005), indican que el nivel socioeconómico sería responsable de al menos parte de los resultados encontrados, observándose que a mayor pobreza mayor cantidad de síntomas de asma $^{35}$.

La evaluación de la epidemiología nacional de la artritis reumatoide es limitada, debido al escaso número de publicaciones. Sólo existen datos hasta la década 1980-89, lo que impide establecer la tendencia durante el periodo transicional que vivió el país durante las últimas décadas. Es importante recalcar que durante nuestra búsqueda bibliográfica no se encontraron estudios epidemiológicos locales sobre enfermedad de Crohn o esclerosis múltiple, dos patologías habitualmente incluidas en este tipo de análisis.

Los resultados que presentamos nos llevan a las siguientes conclusiones: aunque los datos disponibles no son óptimos, sugieren que en Chile se observa el cambio en el patrón epidemiológico de enfermedades ya descrito en países desarrollados. Segundo, la evidencia reciente de la literatura general y nacional apoyan la teoría de la higiene en la aparición de atopia, pero no en el aumento observado de síntomas de asma. Tercero, es urgente que la comunidad médica nacional tome conciencia de la necesidad de producir estudios locales, que permitan establecer los patrones de enfermedad en el país; esto es indispensable para proponer acciones efectivas que mejoren la salud de nuestra población. 


\section{REFERENCIAS}

1. BACH JF. The effect of infections on susceptibility to autoimmune and allergic diseases. N Engl J Med 2002; 347: 911-20.

2. Kilpi T, Kero J, Jokinen J, Syrjanen R, Takala AK, Hovi $\mathrm{T}$ eT AL. Common respiratory infections early in life may reduce the risk of atopic dermatitis. Clin Infect Dis 2002; 34: 620-6.

3. Leibowitz U, Antonovsky A, Medalie JM, Smith HA, HALPERN L, ALTER M. Epidemiological study of multiple sclerosis in Israel. II. Multiple sclerosis and level of sanitation. J Neurol Neurosurg Psychiatry 1966; 29: 60-8.

4. Strachan DP. Hay fever, hygiene, and household size. BMJ 1989; 299: 1259-60.

5. Rautava S, Ruuskanen O, Ouwehand A, Salminen S, Isolauri $\mathrm{E}$. The hygiene hypothesis of atopic disease-an extended version. J Pediatr Gastroenterol Nutr 2004; 38: 378-88.

6. Kemp A, BJorksten B. Immune deviation and the hygiene hypothesis: a review of the epidemiological evidence. Pediatr Allergy Immunol 2003; 14: 74-80.

7. Albala C, VIo F. Epidemiological transition in Latin America: the case of Chile. Public Health 1995; 109: 431-42.

8. Valenzueia P, Gómez G, Galieguilos F. Prevalencia del asma bronquial en escolares de Santiago, Chile. Rev Méd Chile 1981; 109: 259-66.

9. Mallol J, Cortez E, Amarales L, Sánchez I, Calvo M, Soto S ET AL. Prevalencia del asma en escolares chilenos. Estudio descriptivo de 24.470 niños. ISAAC-Chile. Rev Méd Chile 2000; 128: 279-85.

10. Medina E, KaempFrer AM. Morbilidad y atención médica en el gran Santiago. Rev Méd Chile 1979; 107: 155-68.

11. Medina E, Kaemprfer AM, Cornejo E, Hernández E, WALL V. Características y manejo de la morbilidad en Santiago en 1993. Rev Méd Chile 1994; 122: 1421-7.

12. Gómez-Carpio M, Arab P, Medina E, Saffie F, GoRDILO H. Epidemiología de la artritis reumatoidea en Chile. Rev Méd Chile 1966; 94: 315-22.

13. Medina E, KaempfFer AM, Cumsile F, Medina R. Encuesta de morbilidad y atención médica. Santiago. Bol Oficina Sanit Panam 1987; 102: 594605.

14. Carrasco E, Pérez-Bravo F, Dorman J, Mondragón A, SANTOS JL. Increasing incidence of type 1
Diabetes in population from Santiago of Chile: Trends in a period of 18 years (1986-2003). Diabetes Metab Res Rev 2006; 22: 34-7.

15. Joussemet M, Depaquit J, Nicand E, Mac Nab C, Meynard JB, Teyssou R et al. Fall in the seroprevalence of hepatitis A in French youth. Gastroenterol Clin Biol 1999; 23: 447-5.

16. AbiRu N, Kawasaki E, Eguch K. Current knowledge of Japanese type 1 diabetic syndrome. Diabetes Metab Res Rev 2002; 18: 357-66.

17. BACH JF. Insulin-dependent diabetes mellitus as an autoimmune disease. Endocr Rev 1994; 15: 516-42.

18. Skadhauge LR, Christensen K, Kyvik KO, Sigsgaard T. Genetic and environmental influence on asthma: a population-based study of 11,688 Danish twin pairs. Eur Respir J 1999; 13: 8-14.

19. Bodansky HJ, Staines A, Stephenson C, Haigh D, CARTwRIGHT R. Evidence for an environmental effect in the aetiology of insulin dependent diabetes in a transmigratory population. BMJ 1992; 304: 1020-2.

20. Robinson DS, Hamid Q, Ying S, Tsicopoulos A, Barkans J, Bentley AM et al. Predominant TH2like bronchoalveolar T-lymphocyte population in atopic asthma. N Engl J Med 1992; 326: 298-304.

21. LeUNG DY, BIEBER T. Atopic dermatitis. Lancet 2003; 361: 151-60.

22. HuRTADO A, Johnson RJ. Hygiene hypothesis and prevalence of glomerulonephritis. Kidney Int Suppl 2005; (97): S62-7.

23. McGuirk P, Higgins SC, Miшs KH. Regulatory cells and the control of respiratory infection. Curr Allergy Asthma Rep 2005; 5: 51-5.

24. Danese S, SAns M, Fiocchi C. Inflammatory bowel disease: the role of environmental factors. $\mathrm{Au}$ toimmun Rev 2004; 3: 394-400.

25. Tetteh JK, Addae MM, Ishiwada N, Yempewu SM, Yamaguchi S, Ofori-Adjei D et al. Plasma levels of Th1 and Th2 cytokines in Ghanaian children with vaccine-modified measles. Eur Cytokine Netw 2003; 14: 109-13.

26. McIntire JJ, Umetsu SE, Macaubas C, Hoyte EG, Cinnioglu C, Cavali-Sforza L et al. Immunology: hepatitis A virus link to atopic disease. Nature 2003; 425: 576.

27. Malik M, Bakir A, SaAb BA, Roglic G, King H. Glucose intolerance and associated factors in the multi-ethnic population of the United Arab Emirates: results of a national survey. Diabetes Res Clin Pract 2005; 69: 188-95. 
28. Wild S, Roglic G, Green A, Sicree R, King H. Global prevalence of diabetes: estimates for the year 2000 and projections for 2030. Diabetes Care 2004; 27: 1047-5.

29. Carrasco E, Pérez-Bravo F, Santos JL, López G, Calvilíán M, WolfF C et al. One of the lowest validated incidence rates of insulin dependent diabetes mellitus in the Americas: Santiago, Chile. Diabetes Res Clin Pract 1996; 34: S153-7.

30. Serrano-Ríos M, Goday A, Martínez Larrad T. Migrant populations and the incidence of type 1 diabetes mellitus: an overview of the literature with a focus on the Spanish-heritage countries in Latin America. Diabetes Metab Res Rev 1999; 15: 113-32.

31. Larenas G, Montecinos A, Manosalva M, Barthou M, VIDAL T. Incidence of insulin-dependent diabetes mellitus in the IX region of Chile: ethnic differences. Diabetes Res Clin Pract 1996; 34: S147-51.

32. Malol J, Sole D, Asher I, Clayton T, Stein R, SotoQuiroz M. Prevalence of asthma symptoms in Latin America: the International Study of Asthma and Allergies in Childhood (ISAAC). Pediatr Pulmonol 2000; 30: 439-44.

33. ANDERSON HR. Air pollution and trends in asthma. CIBA Foundation Symposium 1997; 206: 190-202.

34. VALDIVIA G. Asma bronquial y enfermedades atópicas como problema emergente de Salud Pública: nuevas hipótesis etiológicas. La experiencia de sociedades desamolladas. Rev Méd Chile 2000; 128: 339-46.

35. Corvalán C, Amigo H, Bustos P, Rona RJ. Socioeconomic Risk Factors for Asthma in Chilean Young Adults. Am J Public Health 2005; [Epub ahead of print]. 